\title{
Ultra-Widefield Fluorescein Angiography as a Predictor for Visual Outcomes in Retinal Vein Occlusion
}

\author{
Clayton Tsang ${ }^{1}$, Amir Hajrasouliha², Sunu Mathew ${ }^{2}$ \\ ${ }^{1}$ Indiana University School of Medicine, ${ }^{2}$ Glick Eye Institute, Indiana University School of \\ Medicine
}

Background and purpose: Retinal vein occlusion (RVO) is the second most common cause of vascular disease leading to visual impairment. Fluorescein angiography (FA), which can visualize areas of vascular non-perfusion, has been used historically to evaluate RVO. Recent advent of ultra-widefield imaging (UWFA) has extended the ability to visualize larger retinal areas, yet the prognostic use is unclear. A retrospective case series study was conducted to better characterize the link between ischemia and visual acuity (VA) outcomes using UWFA. Experimental method: The study used retrospective analysis of patients with RVO from September 2017 until June 2020. UWFA images of patients with RVO were analyzed by correlating VA to the surface area of retinal nonperfusion (NP) or the presence of macular ischemia (MI). VA was recorded at time of UWFA and at follow-up (average 4.7 months) and classified as ischemic if Snellen acuity was 20/200 or worse. NP surface area was measured using Image $(\mathrm{NIH}$, Bethesda, MD). Eyes with abnormal and ischemic foveal avascular zones were categorized as having MI. Results: 13 eyes met inclusion criteria for the study. At initial presentation, ischemic eyes had greater NP compared to nonischemic eyes $(p=0.02)$. At followup, eyes with ischemia had greater NP on their original UWFA compared to nonischemic eyes $(p=0.03)$. When comparing eyes with and without MI, there were no significant differences in VA at initial presentation $(p=0.30)$ or at follow-up $(p=0.44)$. Conclusion and Implications: UWFA is important in the prognosis of RVO patients as visual outcomes correlate to the NP of the entire retina. The presence of $\mathrm{Ml}$ alone was not successful in predicting visual outcomes. This suggests that UWFA is a more powerful diagnostic method compared to traditional FA techniques, which cannot identify peripheral nonperfusion. Future work will center around analyzing other retinal imaging methods and comparing prognostic efficacy. 